\title{
Oropharyngeal (p16-Negative) Cancer pT4b TNM Finding v8
}

National Cancer Institute

\section{Source}

National Cancer Institute. Oropharyngeal (p16-Negative) Cancer pT 4b TNM Finding v8. NCI Thesaurus. Code C132925.

Oropharyngeal (p16-negative) cancer with very advanced local disease. T umor invades lateral pterygoid muscle, pterygoid plates, lateral nasopharynx, or skull base or encases carotid artery. (from AJCC 8th Ed.) 